\title{
One-year results of transcatheter treatment of severe tricuspid regurgitation using the edge-to-edge repair technique
}

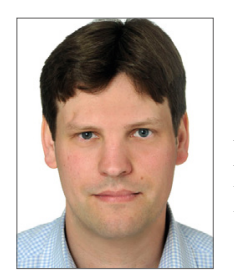

\author{
Daniel Braun ${ }^{1 *}$, MD; Michael Nabauer ${ }^{1}, \mathrm{MD}$; Mathias Orban ${ }^{1,2}, \mathrm{MD}$; Andrea Englmaier ${ }^{1}, \mathrm{MSc}$; \\ Diana Rösler ${ }^{1}, \mathrm{RN}$; Christian Hagl ${ }^{3}$, MD; Steffen Massberg ${ }^{1,2}$, MD; Jörg Hausleiter ${ }^{1}$, MD
}

\begin{abstract}
1. Medizinische Klinik und Poliklinik I, Klinikum der Universität München, Munich, Germany; 2. Munich Heart Alliance, Munich, Germany; 3. Herzchirurgische Klinik und Poliklinik, Klinikum der Universität München, Munich, Germany
\end{abstract}

\section{Introduction}

Transcatheter edge-to-edge repair has been successfully used for the treatment of inoperable patients with severe tricuspid regurgitation (TR). Observational studies were able to demonstrate favourable acute and short-term outcomes ${ }^{1-3}$. However, given the novelty of this treatment approach, no long-term data are available. Here, we present one-year follow-up data of 24 consecutive patients treated with the edge-to-edge repair technique (MitraClip ${ }^{\mathbb{B}}$; Abbott Vascular, Santa Clara, CA, USA). The acute and early outcome results of the first 18 patients have been reported previously ${ }^{1}$.

\section{Methods}

Patients were treated for predominantly secondary TR from March until September 2016 at the Munich University Hospital. Clips were implanted using a modified steering technique as previously described". TR reduction was attempted by applying the "triple orifice technique", where clips are placed centrally between the anterior and septal as well as between the posterior and septal tricuspid leaflets. Alternatively, the "bicuspidalisation technique" was applied, where clips are placed between the anterior and septal tricuspid leaflets.

Primary endpoints were defined as survival, TR grade as well as New York Heart Association (NYHA) functional class at 12-month follow-up. Secondary endpoints were defined as distance in the sixminute walk test (6-MWT), level of N-terminal pro-brain natriuretic peptide (NT-proBNP), quality of life as expressed by the Minnesota Living with Heart Failure Questionnaire (MLHFQ) score, as well as echocardiographic parameters of right ventricular function.

\section{Results}

Mean patient age was $79 \pm 7$ years with a mean STS score of $6 \pm 6 \%$. Eight patients were treated for isolated TR, while 16 patients were treated for combined TR and MR in a single procedure. Patients were treated with systolic pulmonary artery pressures ranging from normal to a maximum of $66 \mathrm{mmHg}$ (mean systolic transtricuspid gradient: $38 \pm 13 \mathrm{mmHg}$ ). Mean LVEF at baseline was $46 \pm 15 \%$. Mean right ventricular function was impaired as expressed by a tricuspid annular plane systolic excursion (TAPSE) of $16 \pm 4 \mathrm{~mm}$ and a mean right ventricular ejection fraction (RVEF) of $38 \pm 8 \%$ at baseline.

Acute procedural success (TR $\leq 2$ ) was achieved in $96 \%$ of patients $(23 / 24)$. A total of 56 clips were implanted (mean: $2.3 \pm 0.7$; median: two per patient). While $42 \%$ of patients were treated using the triple orifice technique, $58 \%$ of patients were treated using the bicuspidalisation technique. We did not observe any procedure-related adverse events. 
During one-year follow-up nine patients died (37.5\%). Causes of death included end-stage heart failure in four patients, sepsis in two patients, STEMI in one patient and unknown causes in two patients.

The rate of patients with TR $\leq 2$ improved from $4 \%$ (1/24) at baseline to $87 \%$ of the surviving patients $(13 / 15)$ at one-year follow-up $(p<0.001)$ (Figure 1). None of the patients with recurrent severe TR underwent surgical repair. However, two patients underwent a second edge-to-edge procedure due to recurrent severe TR. In one patient treated with three clips into the anteroseptal commissure, recurrent severe TR occurred due to single leaflet clip detachment from the septal leaflet of the most inferiorly placed clip one month following the procedure. The patient was treated by placing another clip into the posteroseptal commissure resulting in an overall reduction of one TR grade. In another patient, recurrent severe TR was observed in the posteroseptal commissure after isolated treatment of the anteroseptal commissure with two clips about two months following the procedure. The patient was successfully treated by placing another clip into the posteroseptal commissure.

In all patients treated simultaneously for severe TR and MR, the grade of MR was reduced to $\leq 2$ at one-year follow-up (12/12).

Transcatheter treatment was associated with significant clinical benefit as evident from an improvement in NYHA functional class. The rate of patients in NYHA $\leq$ II improved from $0 \%$ $(0 / 24)$ at baseline to $67 \%$ of the surviving patients $(10 / 15)$ at oneyear follow-up $(p<0.001)$ (Figure 2). Clinical benefit in surviving patients treated for isolated TR appeared comparable to that in patients treated for TR and MR ( $p>0.99)$. Concerning optimal medical therapy, diuretic therapy remained unchanged in $38 \%$ of patients, was decreased in $31 \%$ of patients and was increased in $31 \%$ of patients over the course of one year.

In a paired analysis there was a trend for improvement in the six-minute walking distance from $205 \pm 136 \mathrm{~m}$ at baseline to $268 \pm 134 \mathrm{~m}$ at one-year follow-up $(\mathrm{p}=0.28)$. The level of NT-proBNP was reduced significantly from $18,573 \pm 39,889 \mathrm{pg} / \mathrm{ml}$ at baseline to $6,483 \pm 8,811 \mathrm{pg} / \mathrm{ml}$ at one-year follow-up $(\mathrm{p}=0.03)$. In line with these results, there was a trend for improvement in quality of life as expressed by a reduction in the MLHFQ score from $35 \pm 18$ to $27 \pm 16(\mathrm{p}=0.12)$.

Right ventricular function remained stable as expressed by a TAPSE of $15 \pm 3$ and $15 \pm 4 \mathrm{~mm}$ as well as an RVEF of $35 \pm 8$ and $31 \pm 6 \%$ at baseline and follow-up, respectively.

\section{Discussion}

This study demonstrates the one-year durability of edge-toedge repair for the treatment of inoperable patients with severe TR. TR reduction appeared stable over the course of one year. Furthermore, clinical benefit was preserved as expressed by a persistent improvement in NYHA functional class. The positive effect of percutaneous TR treatment was further emphasised by a significant decrease in the level of NT-proBNP. Given the small size of the study population, improvement in six-minute walking distance and reduction in the MLHFQ score did not reach statistical significance. In addition, the initial restriction of applying this repair to patients qualifying for "compassionate use" limits the survival prospects, also evident by significant mortality in this study. It is conceivable that an earlier TR treatment in the heart failure process might result in a better survival outcome.
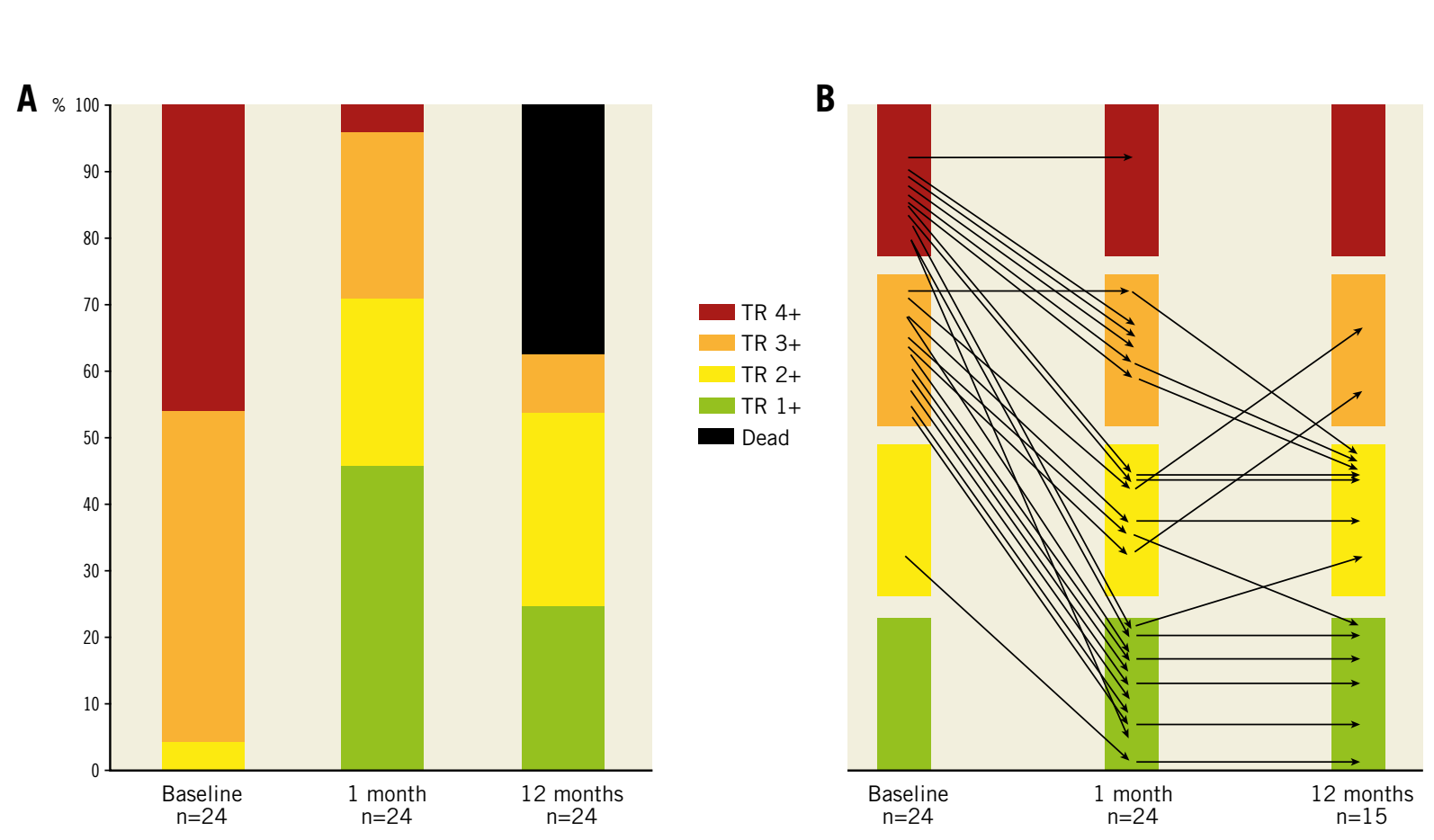

Figure 1. Development of TR. TR grade at baseline vs. one-month and 12-month follow-up. A) Distribution of TR grade including deceased patients. B) Development of TR for each individual patient. 

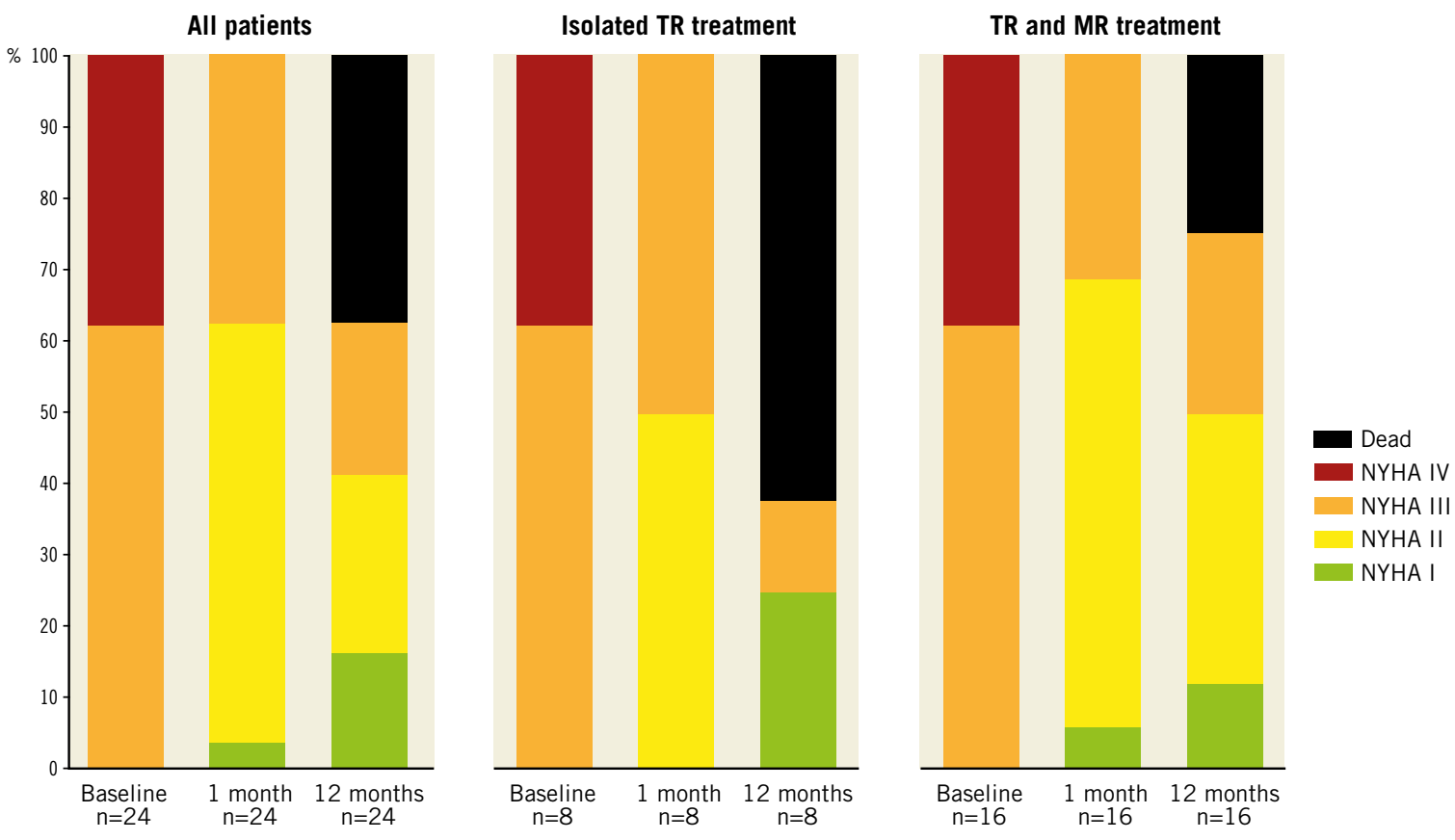

Figure 2. Changes in NYHA functional class. NYHA status at baseline vs. one-month and 12-month follow-up in all patients, in patients treated for isolated TR and in patients with combined TR and MR treatment.

It is reassuring that parameters of right ventricular function were stable over one year, given the arguments that TR reduction would have detrimental effects on right ventricular function due to an increase in afterload. Further larger studies are needed to evaluate a potential positive long-term effect on right ventricular function. For the first time, the low risk of this interventional procedure allows study of the effects of TR reduction without the distortion of operative mortality.

\section{Limitations}

The limitations of this study are the low number of patients as well as the combined treatment of severe TR and MR in two thirds of patients. TR improvement as well as clinical benefit might be partly due to successful MR treatment in the latter patients.

\section{Conclusion}

The preliminary echocardiographic and clinical results reported here encourage further studies on the use of percutaneous edge-toedge repair in inoperable patients with severe TR.

\section{Impact on daily practice}

Patients with severe TR frequently present with severe rightsided heart failure despite optimal medical therapy. Transcatheter repair of severe TR using the edge-to-edge repair technique appears to be durable and associated with maintained clinical improvement over the course of one year in patients ineligible for cardiac surgery.

\section{Funding}

Our institution has received a research grant from Abbott Vascular, not related to this study.

\section{Conflict of interest statement}

D. Braun and M. Nabauer have received speaker honoraria from Abbott Vascular. J. Hausleiter has received speaker honoraria from Abbott Vascular and Edwards Lifesciences. The other authors have no conflicts of interest to declare.

\section{References}

1. Braun D, Nabauer M, Orban M, Orban M, Gross L, Englmaier A, Rosler D, Mehilli J, Bauer A, Hagl C, Massberg S, Hausleiter J. Transcatheter treatment of severe tricuspid regurgitation using the edge-to-edge repair technique. EuroIntervention. 2017;12:e1837-44.

2. Nickenig G, Kowalski M, Hausleiter J, Braun D, Schofer J, Yzeiraj E, Rudolph V, Friedrichs K, Maisano F, Taramasso M, Fam NP, Bianchi G, Bedogni F, Denti P, Alfieri O, Latib A, Colombo A, Hammerstingl C, Schueler R. Transcatheter Treatment of Severe Tricuspid Regurgitation with the Edge-to-Edge: MitraClip Technique. Circulation. 2017;135:1802-14.

3. Orban M, Besler C, Braun D, Nabauer M, Zimmer M, Orban M, Noack T, Mehilli J, Hagl C, Seeburger J, Borger M, Linke A, Thiele H, Massberg S, Ender J, Lurz P, Hausleiter J. Sixmonth outcome after transcatheter edge-to-edge repair of severe tricuspid regurgitation in patients with heart failure. Eur $J$ Heart Fail. 2018;20:1055-62. 